\title{
Added insight from image-based wettability characterization
}

\author{
Rudolf Held $^{1, *}$, Norbert Schleifer ${ }^{1}$, Luis Genolet ${ }^{1}$, Andrew Fogden ${ }^{1}$ \\ ${ }^{1}$ Wintershall Holding GmbH, Germany
}

\begin{abstract}
Microtomographic rock and fluid imaging under in-situ conditions is applied for reservoir wettability characterization. The investigation entails careful sample preparation and cleaning of mini-plugs, operation with reservoir fluids, wettability restoration, centrifuge wettability testing cycles, repeated sample scanning and image analysis, parametrization of wettability and digital rocks simulation for input into reservoir modeling. The results are compared to conventional Amott testing performed in core laboratories. Determination of saturations from image analysis, instead of centrifuge production, allows the use of stock tank crude, rather than exchanged mineral oil. Doping of the synthetic formation water (here with $1 \mathrm{M}$ sodium iodide) was applied for enhancement of the X-ray contrast. The digital imaging workflow offers insight on the liquid distributions from the plug scale down to the pore-scale, linked to applied pressure gradients and resulting pore fluid occupancies in the sequence of displacement states. An example is given with the investigation of a North-German oil field, where the image-based workflow led to a revised view of the reservoir conditions for spontaneous imbibition and drainage, and the overall wetting behavior.
\end{abstract}

\section{Introduction}

A conventional study of reservoir wettability relies on standard, well-established, laboratory testing procedures, such as Amott [1] or USBM [2] index determination or new methods based on cutting-edge approaches as the one proposed by Strand et al. [3]. The former methods macroscopically measure spontaneous water imbibition and/or spontaneous oil drainage to characterize the wetting state of core plugs. The latter methods compare the fractional pore surface area covered by water or oil. Cumulative volumes of produced oil, respectively produced water or adsorbing / non-adsorbing tracers, are used as semi-quantitative, mostly qualitative, indicators. The wetting behavior of a reservoir derives from the electrostatic interaction of surface active compounds in crude oil with the brine/rock system (CBR) [4-5], and consequently, wettability is conceived as an attainable alteration condition of the CBR system and not as a physical property of the rock. The more recent investigations of pore-scale processes and pore fluid distributions via direct imaging aim at a fundamental characterization of reservoir wettability [e.g., 6-8]. The current study employs micro computed tomographic imaging (micro-CT) to relate macroscopically observed production to underlying wetting characteristics and detailed information on saturation distributions.

\footnotetext{
* corresponding author: rudolf.held@wintershall.com
}

The studied North-German oil field is a shallow, weakly consolidated mid- to coarse- grained sandstone reservoir of Upper Valangian, Cretacious age. Reservoir permeability can reach up to several Darcy, with porosities from 18 to $30 \%$. The field was discovered in the early 1950 s and currently still produces, with a high water cut of $96 \%$. Based on numerous laboratory studies concentrating only on half-Amott wettability tests and delivering an Amott water index $\alpha_{w}$ greater than 0.5 for aged, as well as cleaned, state samples, the reservoir is historically assumed to be water-wet.

A recent core analysis study was performed between 2012 and 2014 on newly drilled core material, covering wettability measurements on aged samples, and for the first time covering a full Amott test cycle. The measured water index $\alpha_{w}$, confirmed the legacy data. As the oil index $\alpha_{o}$, obtained was greater than $\alpha_{w}$, the resulting combined Amott-Harvey index falls between -0.1 and 0.3 , characterizing the reservoir as mixed-wet towards slightly oil-wet. As these findings contradict the existing view on the reservoir conditions, digital rock analyses were initiated to further investigate the laboratory results.

\section{Image-based wettability characterization}

For this latest laboratory study, a set of core plugs (1.5" diameter) from three wells of the reservoir was restored for combined USBM/Amott wettability and capillary pressure determination. The workflow involved primary 
drainage and aging in stock-tank crude oil (STO), exchange of the STO for laboratory mineral oil, forced imbibition of synthetic formation water (SFW) and forced secondary drainage of mineral oil via centrifugation.

Additional plugs, of 44-48 mm length, that had not been used in these SCAL studies, were selected as candidates for the image-based wettability study. MicroCT scans of the plugs revealed that all comprised a moderately heterogeneous packing of poorly-sorted quartz grains, sporadically interspersed with clusters of dense mineral cement, and with preparation damage in the vicinity of the plug ends. From the best candidate plug, a sub-plug of $12 \mathrm{~mm}$ diameter was cored to achieve (1) sufficiently small voxel resolution of the pores and throats over a representative lateral field of view, and (2) a sufficiently tall and robust sub-plug that would remain intact through coring and centrifugation at capillary pressures $\mathrm{P}_{\mathrm{c}}$ close to the standard plug investigation.

The weakly consolidated sandstone plug was immersed under ambient pressure in pre-cleaned, molten paraffin wax, which was then hardened at $4^{\circ} \mathrm{C}$. The subplug was cored from the wax-supported plug with water as lubricant, and then sleeved in fluoropolymer heatshrink with a steel mesh at top and bottom, and flowthrough cleaned with heptane to remove residual wax. The cleaned, dried sub-plug was scanned by helical micro-CT at $100 \mathrm{kV}$ with $5.0 \mu \mathrm{m}$ voxel size, covering nearly the entire height of $31.7 \mathrm{~mm}$. A central longitudinal slice of the dry-state tomogram is shown in Figure 1a, verifying the relative homogeneity of the sample and the adequate sample size.

The sub-plug was then re-scanned at multiple stages of its laboratory restoration and centrifuge wettability testing. Forced displacement steps were performed in a rock ultra-centrifuge (Beckman L8) for 30 hours at $40^{\circ} \mathrm{C}$. Sub-plug tomograms were acquired at the following stages:

(SAT) Brine saturation: Using doped SFW with $1.0 \mathrm{M}$ of sodium chloride substituted for $1.0 \mathrm{M}$ of sodium iodide (yielding density of $1.20 \mathrm{~g} / \mathrm{cm}^{3}$ ); Primary drainage: Centrifugation in STO (density $0.8928 \mathrm{~g} / \mathrm{cm}^{3}$ ) at $3500 \mathrm{rpm}$ $\left(\mathrm{P}_{\mathrm{c}}=96 \mathrm{kPa}\right)$;

(AG) Aging: Statically, in STO for 28 days at $54^{\circ} \mathrm{C}$;

(FI_0) Spontaneous imbibition: Immersion in doped SFW for 14 days at $54^{\circ} \mathrm{C}$;

(FI_1) Forced imbibition: Centrifugation in doped SFW at $450 \mathrm{rpm}\left(\mathrm{P}_{\mathrm{c}}=-3.0 \mathrm{kPa}\right)$;

(FI_2) Forced imbibition: Centrifugation in doped SFW at $3650 \mathrm{rpm}\left(\mathrm{P}_{\mathrm{c}}=-198 \mathrm{kPa}\right)$;

(SD_0) Spontaneous secondary drainage: Immersion in STO for 14 days at $54^{\circ} \mathrm{C}$;

(SD_1) Forced secondary drainage: Centrifugation in $\mathrm{STO}$ at $400 \mathrm{rpm}\left(\mathrm{P}_{\mathrm{c}}=1.3 \mathrm{kPa}\right)$.

Each of these eight tomograms of the sub-plug was registered into geometric alignment in 3D with the drystate tomogram, which verified that grain movement during these steps was not perceivable. Tomogram grayscales were then linearly normalized to match between the different states. The dry-state tomogram (Figure 1a) was then segmented using a converging active contours algorithm into three phases: (1) resolved pores (dark voxels), (2) resolved grains (bright voxels), and (3) remaining fraction, comprising sub-resolution features and partial volume effects.

The porosity at each voxel of this intermediate phase was quantified from the difference tomogram of the brinesaturated state minus the dry state, in which the registered, normalised mineral contributions cancel so that the signal at each voxel is directly related to the volume of doped SFW filling the pore space there. This results in the quantitative porosity map of the sub-plug, where black is $100 \%$ resolved porosity, white is $100 \%$ solid mineral, and the intermediate grayscales represent sub-resolution volumes with a porosity value between $0-100 \%$ in linear relation to the voxel grayscale. This calibrated difference imaging workflow strongly reduces the impact of image resolution limitations on the presented quantitative fluid contents.

The calibrated porosity map forms the basis of all saturation calculations on the series of oil-containing tomograms collected during the wettability workflow. In these states, STO is readily segmented, as it is much darker than the doped SFW. The segmented oil phase was then overlain onto the porosity map to quantify the oil occupancy of resolved and sub-resolution pore voxels; the brine phase thus corresponds to the remainder of pore space.

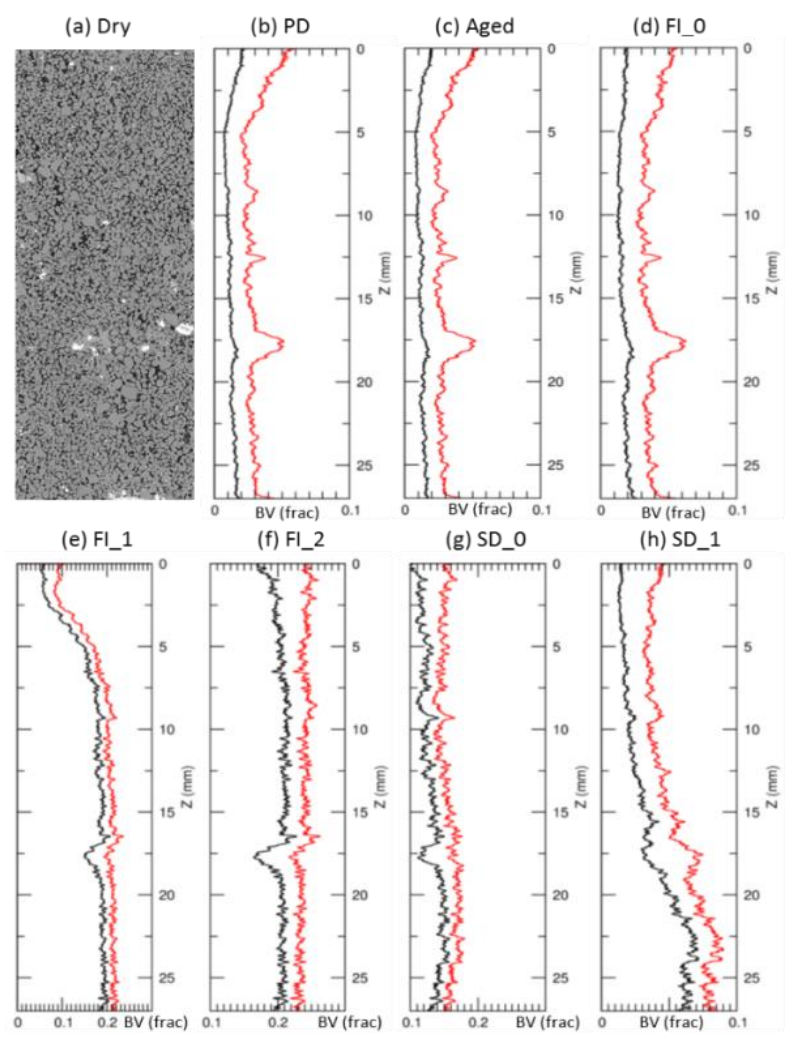

Fig. 1. Central longitudinal slice $(10.0 \mathrm{~mm} \times 27.5 \mathrm{~mm})$ of the $12 \mathrm{~mm}$ diameter sub-plug in (a) dry state and (b-h) longitudinal profiles of SFW bulk volume fraction for $\mathrm{PD}=$ primary drainage, $\mathrm{FI}=$ forced imbibition, and $\mathrm{SD}=$ secondary drainage. Black curves are the volume contribution from resolved pores and red curves represent total values from resolved and subresolution pores. 
(a) Dry

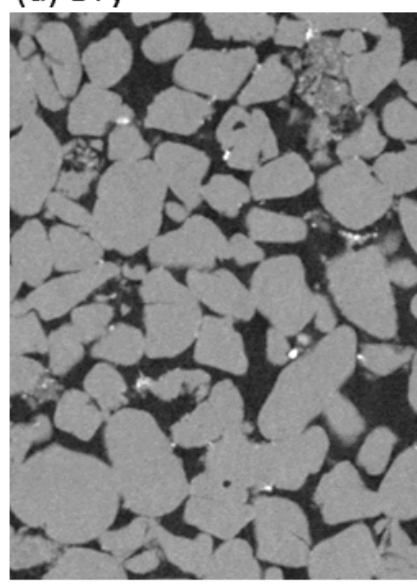

(d) $\mathrm{Fl}$

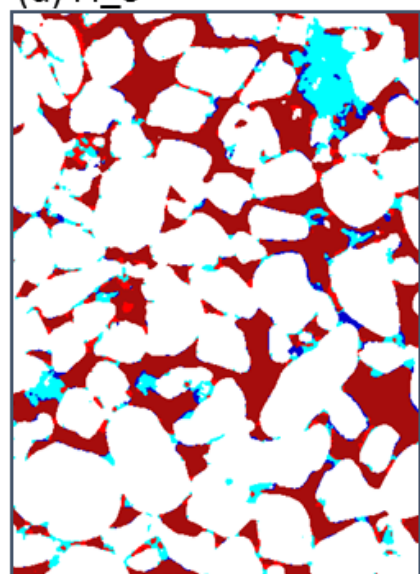

(g) SD_0

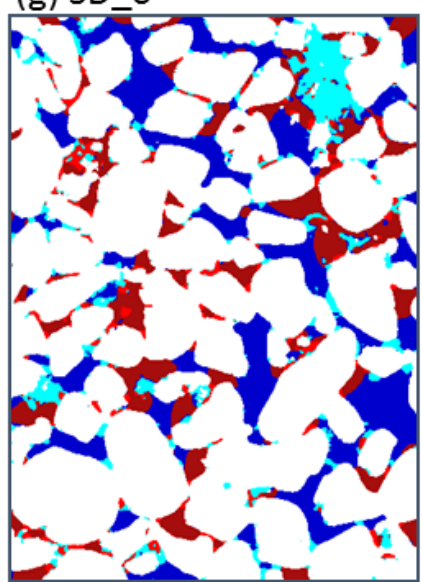

(b) PD
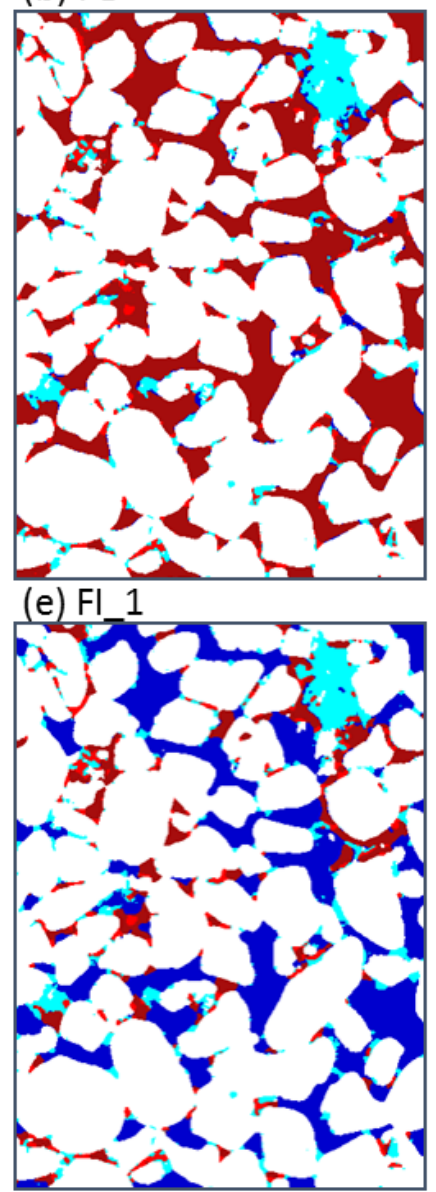

(h) SD_1

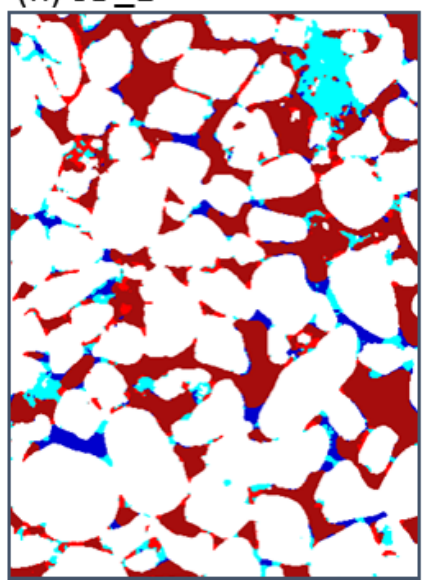

(c) Aged

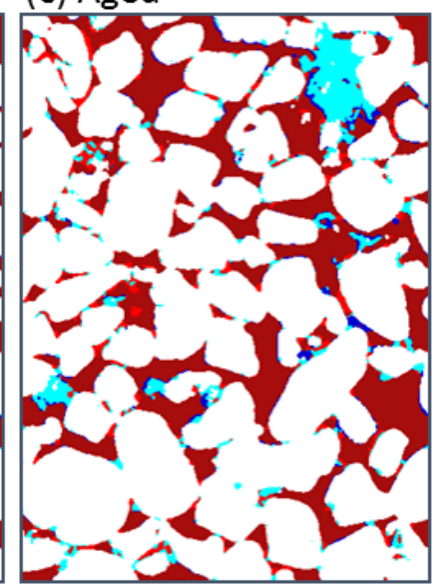

(f) $\mathrm{FI} 2$
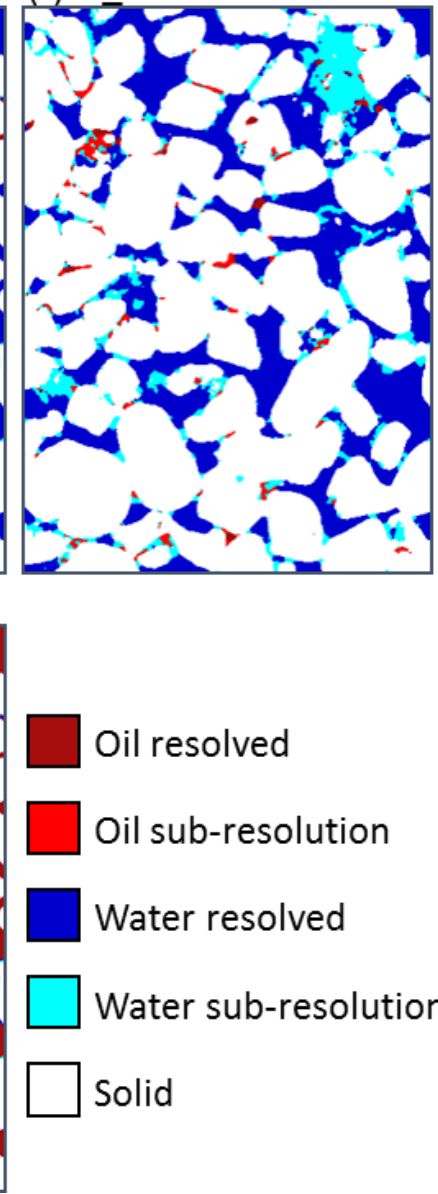

Oil resolved

Oil sub-resolution

Water resolved

Water sub-resolution

Solid

Fig. 2. Zoom into sub-area $(1.9 \mathrm{~mm} \times 2.6 \mathrm{~mm})$ of the sub-plug tomograms corresponding to Figure 1, illustrating varying pore fluid occupancy, as well as showing the segmentation color legend.

\section{Observed fluid distributions}

The dry-state tomogram of the sub-plug, represented by the longitudinal slice in Figure 1a and the sub-area in Figure 2a, shows the range of grain sizes and intergranular pores and throats. Quartz grains are abundant, along with weathered and lithic grains and agglomerates that comprise a mixture of clay minerals. Denser minerals, likely siderite cement and pyrite, are relatively sparse in this sample. The segmented porosity map of the sub-plug gives a resolved porosity of 20.7 volume $\%$ at voxel size of $5.0 \mu \mathrm{m}$, while sub-resolution porosity amounts to $3.9 \%$. This yields an image-based total porosity of the sub-plug of $24.6 \%$. Thus, the majority of the intergranular pore space, i.e. pore space contributing to flow in the sample, could be resolved. Non-resolved porosity is present within the lithic grains and clay regions; the remainder of this sub-resolution porosity stems from partial volume effects at grain edges. The total porosity value of the sub-plug is somewhat lower than the Helium porosity measured at $26.9 \%$ on the closest neighboring core plug. This may be 
attributed to sample variability in local porosities, or partially to the exclusion of identified damaged plug ends in the image-based workflow.

The sequence of oil-containing states of the sub-plug was acquired in steps 1-7 of the wettability testing workflow. Their tomograms after registration to the dry state and segmentation are represented by the longitudinal profiles of SWF bulk volume fractions in Figures $1 \mathrm{~b}-\mathrm{h}$ (averaged over each tomogram cross-sectional slice), and by the same sub-area in Figures $2 \mathrm{~b}-\mathrm{h}$ (illustrating porescale details). The top of the profiles in Figure 1 is the inner end of the sub-plug in the centrifuge during forced displacement, or the top of the sub-plug when standing immersed for spontaneous displacement. The direction of gravitational force is thus always downwards. Blue and brick red in the color segmentation in Figure 2 correspond to water (doped SFW) and oil in resolved pores, while cyan and bright red represent water and oil in subresolution porosity. White is resolved solid mineral. Within voxels of the cyan and bright red phases, water and oil only occupy the pore volume fraction given by the porosity map. Table 1 lists the tomogram average of the bulk volume fractions of each of these four fluid phases in the seven oil-containing states.

After primary drainage (Figure $1 b$ and $2 b$ ), average water saturation in the sub-plug is $S_{w}=0.123$. The subresolution porosity within clay aggregates mainly stays water-filled. Water is also observed as necks bridging intergranular throats and as films lining rougher grain surfaces, whereas the vast majority of the pore space is oil-filled. This oil occupancy includes most intergranular slot pores, throats and corners, often segmented as subresolution porosity (bright red) due to partial volume effects. Water saturation is slightly higher near the bottom in Figure 1b, where capillary pressure was lowest in the centrifuge. The increase in water saturation at around onethird of the sub-plug height corresponds to a local siderite band with adjacent non-resolved porosity. The fluid occupancy after ageing (Figure 1c and 2c) is nearly identical to the primary drainage state with an imagebased average saturation of $S_{w}=0.120$. Ageing does not give rise to much resolvable change in menisci, nor to self-driven advance of oil into porosity associated with lithic grains and clays. Wettability alteration cannot be directly visualized, since the underlying brine thin-film rupture and adsorption of oil polar components lie well below the imaging resolution.

Spontaneous imbibition at zero applied $\mathrm{P}_{\mathrm{c}}$ (FI_0) in Figure $1 \mathrm{~d}$ and $2 \mathrm{~d}$ results in a small increase in water in both resolved and sub-resolution pores (equal to $0.4 \% \mathrm{BV}$ in each category in Table 1). This translates to a change in average water saturation to $S_{w}=0.154$. Spontaneous imbibition is due to connected water in the aged state advancing to displace oil from adjacent water-wet locations. The observed imbibition does not extend to displacement of oil from pore bodies, as oil retains sufficient anchoring to grain surfaces.

Forced imbibition at low spin speed (FI_1) already gives a strong overall increase in average water saturation to $S_{w}=0.772$ (cf. Table 1), with a clear gradient along the sub-plug in Figure 1e. Water saturation decreases from bottom to top, especially steeply so towards the inner (top) end in the centrifuge, in response to the decreasing magnitude of capillary pressure. Although it is conceivable that the centrifuge-induced saturation gradient could change by liquid redistribution during scanning, no liquid movement was observed from the radiographs over the 12 -hour scanning duration. The high oil saturation towards the top end is a result of decreased displacement, rather than accumulation. Instances of oil entering and becoming stranded in brine-filled pores during imbibition are rarely detected. Oil is generally displaced from larger pore bodies, but is retained by smaller intergranular pores, corners and slots.

At the highest centrifugation speed of forced imbibition (FI_2 in Figure 1f and 2f), residual oil saturation is uniformly very low at $S_{\text {or }}=0.030$. The capillary pressure gradient is no longer apparent from saturation imaging; even the smallest magnitude of $P_{c}$ at the top end of the sub-plug suffices to displace oil from all but its tightest locations in slot pores.

Spontaneous secondary drainage at zero applied $\mathrm{P}_{\mathrm{c}}$ (SD_0) is substantial (cf. Table 1), yielding an average water saturation of $S_{w}=0.636$ from the tomogram analysis. Figure $1 \mathrm{~g}$ exhibits a slight gradient of decreasing water saturation from bottom to top. This is attributed to gravity and capillary end effects. Oil spontaneously enters small pores and throats (except clay-associated, waterfilled, tight pores) and extends into larger pores to fully or partially displace water, or leave water-filled individual pores or clusters behind. The observed spontaneous secondary drainage state is not as strongly oil-wet as to result in snap-off of brine in the center of pores.

Forced secondary drainage at low spin speed (SD_1) results in further increase in oil saturation to $S_{w}=0.221$. As was also the case for FI_1, water saturation decreases, respectively oil saturation increases, from bottom to top in Figure $1 \mathrm{~h}$ due to the capillary pressure increase in that direction. Oil is observed to advance further into waterfilled pore spaces, either partially near the bottom end or completely towards the top. At voxel resolution, water often appears isolated, remaining in constricted small pores or at grain-to-grain contacts, especially those near brine-filled clay pores. Although phase connectivity may not be directly resolved, related volumetric changes can be quantified.

A higher centrifugation speed of the secondary drainage cycle to lower $S_{w}$ was omitted. 
Table 1. Tomogram-averaged water and oil volume fractions (percentages of total bulk volume) given as resolved (Res), subresolution (Sub-res) pores and total (Tot) for the $12 \mathrm{~mm}$ diameter sub-plug volume in the brine-saturated (SAT) state and the seven oil-containing states in Figure 1 b-h.

\begin{tabular}{|c|r|r|r|r|r|r|}
\hline Fraction & \multicolumn{3}{|c|}{ water } & \multicolumn{3}{c|}{ oil } \\
\hline State & $\begin{array}{c}\varphi_{\mathrm{w}} \text { Res } \\
(\% \mathrm{BV})\end{array}$ & $\begin{array}{c}\varphi_{\mathrm{w}} \text { Sub-res } \\
(\% \mathrm{BV})\end{array}$ & $\begin{array}{r}\varphi_{\mathrm{w}} \text { Tot } \\
(\% \mathrm{BV})\end{array}$ & $\begin{array}{c}\varphi_{\mathrm{o}} \text { Res } \\
(\% \mathrm{BV})\end{array}$ & $\begin{array}{c}\varphi_{\mathrm{o}} \text { Sub-res } \\
(\% \mathrm{BV})\end{array}$ & $\begin{array}{r}\varphi_{\mathrm{o}} \text { Tot } \\
(\% \mathrm{BV})\end{array}$ \\
\hline SAT & 20.7 & 3.9 & $\mathbf{2 4 . 6}$ & & & \\
\hline PD & 1.3 & 1.8 & $\mathbf{3 . 0}$ & 19.5 & 2.1 & $\mathbf{2 1 . 6}$ \\
\hline Aged & 1.3 & 1.7 & $\mathbf{2 . 9}$ & 19.5 & 2.2 & $\mathbf{2 1 . 7}$ \\
\hline FI_0 & 1.7 & 2.1 & $\mathbf{3 . 8}$ & 19.0 & 1.8 & $\mathbf{2 0 . 8}$ \\
\hline FI_1 & 16.5 & 2.5 & $\mathbf{1 9 . 0}$ & 4.3 & 1.3 & $\mathbf{5 . 6}$ \\
\hline FI_2 & 20.4 & 3.4 & $\mathbf{2 3 . 9}$ & 0.3 & 0.4 & $\mathbf{0 . 7}$ \\
\hline SD_0 & 13.0 & 2.6 & $\mathbf{1 5 . 6}$ & 7.7 & 1.3 & $\mathbf{9 . 0}$ \\
\hline SD_1 & 3.5 & 2.0 & $\mathbf{5 . 4}$ & 17.3 & 1.9 & $\mathbf{1 9 . 1}$ \\
\hline
\end{tabular}

\section{Inferred capillary pressure curves}

Figure 3 combines all $\mathrm{P}_{\mathrm{c}}\left(\mathrm{S}_{\mathrm{w}}\right)$ data from the image analysis of the sub-plug and compares these to the results from combined USBM/Amott wettability testing of the closest neighboring plug. For the sub-plug, the seven curve segments are obtained from the longitudinal profiles of total (resolved plus sub-resolution) $S_{\mathrm{w}}$ in Figure 1. The distance along the sub-plug axis is converted to the corresponding $\mathrm{P}_{c}$ value given by the centrifugal force difference. This $\mathrm{P}_{\mathrm{c}}$ calculation used the uncorrected centrifuge equation with distances from the rotation axis to the sub-plug bottom (outer end). $\mathrm{P}_{\mathrm{c}}$ was assumed to be zero at the sub-plug bottom in drainage and sub-plug top in imbibition, located $2.1 \mathrm{~mm}$ below and above the section displayed in Figure 1.

The line intervals of saturation at $\mathrm{P}_{\mathrm{c}}=0$ for spontaneous imbibition and secondary drainage span the variability between slices that arise at various scales: from pore-scale local heterogeneities, such as microporous clusters with high $S_{w}$, to registered gradients along the sub-plug (for SD_0). The mean values of $\mathrm{S}_{\mathrm{w}}$ for FI 0 and SD 0 are indicated by unfilled circles in Figure 3 . For the three curves PD, FI 1 and SD 1, the above-mentioned centrifuge-induced gradients along the sub-plug reveal the trend of the $\mathrm{P}_{\mathrm{c}}\left(\mathrm{S}_{\mathrm{w}}\right)$ relation, with local variability superposed. The observed spike in PD at around $40 \mathrm{kPa}$ in Figure 3 relates to the above-mentioned siderite bandassociated tight porosity in Figure 1b. The curve for FI_2 shows rather uniform saturation distribution of $S_{w}$ along the sub-plug.

These recovered trends from the image-based experiments are compared with the conventional plug measurements included in Figure 3. The conventional laboratory testing substituted spontaneous imbibition and secondary drainage by centrifuge measurements at 300 rpm, i.e., $\mathrm{P}_{\mathrm{c}}=-4.3 \mathrm{kPa}$ and $\mathrm{P}_{\mathrm{c}}=2.1 \mathrm{kPa}$, respectively.
These Pc magnitudes already exceed those for the imaged sub-plug states FI_1 and $\mathrm{SD}_{-} 1$, respectively. The $\mathrm{S}_{\mathrm{w}}$ range of the imaged PD curve from the sub-plug, and the almost superposed AG curve, is lower than the corresponding plug endpoint before aging (PD Meas) and more in line with the saturation point after aging (AG Meas). This significant drop in plug-measured $\mathrm{S}_{\mathrm{wi}}$ seems to be due to water displacement during exchange of mineral oil for STO or during flow-through aging, but does not appear to be a direct consequence of wettability alteration based on the sub-plug results. $\mathrm{S}_{\text {or }}$ from the imaged FI_2 curve is also lower than the corresponding plug endpoint (FI Meas). The measured FI and SD curves are somewhat steeper than the corresponding imaged curves, as noticeable in the expanded plot in Figure 3.

The discrepancies may partially stem from differences in the respective experiments. The $\mathrm{P}_{c}$ range of the plug measurements is broader, owing to the larger plug length (49 $\mathrm{mm}$ ) and fluid density difference (SFW-mineral oil). This limits the scope for measurement at low pressures and weakens the assumption that the lowest spin speeds of forced imbibition and secondary drainage are good approximations to spontaneous recovery, especially for such highly permeable samples of intermediate wettability. Accordingly, the resulting estimates of Amott indices for water and oil in Table 2 are both higher from plug measurement than from sub-plug imaging. However, the combined Amott-Harvey index is similar, and both studies classify the investigated oil/brine/rock system as moderately oil-wet. As the experiments used differing fluid combinations and plug samples, J-Function scaling yields better comparability of the results. Nevertheless, the curves from sub-plug imaging with STO as oil remain slightly flatter over the intermediate saturation interval, suggesting also some variation in the pore size distribution of the two neighboring samples. 

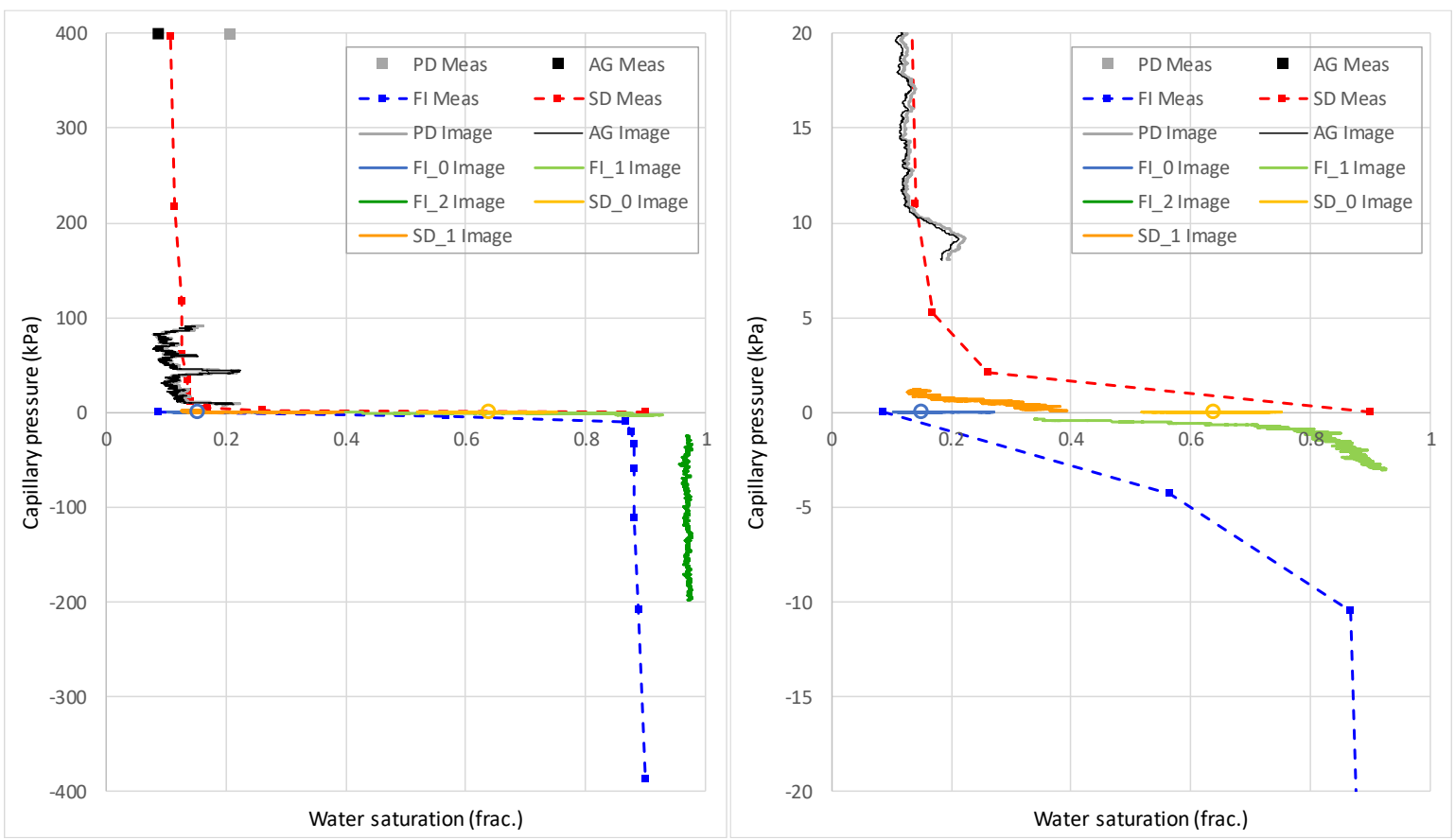

Fig. 3. Plot of water saturation versus capillary pressure from image analysis of the sub-plug (solid curves) and from the traditional laboratory measurement on a nearby plug (Meas, points and dashed curves). The curves in the left plot at low pressures are shown expanded in the right plot.

Table 2. Amott index for water and oil and combined AmottHarvey and USBM indices from Figure 3 for the imaged subplug and measured plug systems, together with their simulations.

\begin{tabular}{|c|r|r|r|r|}
\hline \multirow{2}{*}{$\begin{array}{c}\text { Wettability } \\
\text { Index }\end{array}$} & \multicolumn{2}{|c|}{ Sub-plug } & \multicolumn{2}{c|}{ Plug } \\
\cline { 2 - 5 } & $\begin{array}{c}\text { Image- } \\
\text { based }\end{array}$ & Simulated & Measured & Simulated \\
\hline$\alpha_{w}$ & 0.04 & 0.07 & 0.60 & 0.08 \\
\hline$\alpha_{o}$ & 0.39 & 0.40 & 0.87 & 0.24 \\
\hline I AH $_{\text {AH }}$ & $\mathbf{- 0 . 3 5}$ & $\mathbf{- 0 . 3 3}$ & $\mathbf{- 0 . 2 7}$ & $\mathbf{- 0 . 1 6}$ \\
\hline IUSBM $_{\text {y }}$ & & $\mathbf{- 0 . 6 6}$ & $\mathbf{- 0 . 2 8}$ & $\mathbf{- 0 . 5 6}$ \\
\hline
\end{tabular}

\section{Digital rocks simulation and analysis}

From the image-based porosity map, a pore network was derived for digital rocks simulations. The cylindrical subplug volume was therefore cropped into an rectangular column. The commercial e-Core software [9] was used to extract the pore network representation and perform corresponding numerical simulations of the Amott-tests. Figure 4 displays the simulation of imbibition after primary drainage to $S_{w i}$, and secondary drainage from $S_{o r}$, for the image-based sub-plug (left) and laboratory plug measurements (right). Anchoring of the simulations was made with regard to the saturation endpoints, i.e. $\mathrm{S}_{\mathrm{wi}}, \mathrm{S}_{\mathrm{or}}$, as well as tomogram-averaged saturations at $\mathrm{P}_{\mathrm{c}}=0 \mathrm{kPa}$ for spontaneous imbibition (corresponding to Figure 1d and 2d) and spontaneous secondary drainage (corresponding to Figure $1 \mathrm{~g}$ and $2 \mathrm{~g}$ ). The match to the sub-plug capillary pressure-saturation data required a wider contact angle range with $\theta_{\mathrm{o}}$ from 110 to 160 for the oil during imbibition, compared to a contact angle range $\theta_{\mathrm{o}}$ from 110 to 138 for the core plug measured data.

The wettability indices derived from the digital rock simulation are compiled in Table 2. The agreement of simulated and image-based wettability indices relates to the anchoring. Additionally, a USBM index could be obtained from simulation, indicating with $\mathrm{I}_{\mathrm{USBM}}<\mathrm{I}_{\mathrm{AH}}$ that preferentially small pores are oil-wet in accordance with theoretical investigations [10]. The same holds for the simulated wettability indices for the laboratory core plug, whereas laboratory-reported $\mathrm{I}_{\mathrm{USBM}}$ and $\mathrm{I}_{\mathrm{AH}}$ are nearly equal, implying no pore-size preference for wetting alteration.

The resolution of the acquired 3D tomograms would allow further analysis into the image-recorded contact angles. An extraction of in-situ contact angle ranges [1112] can provide a directly measured indication of the wetting behaviour of the sample and modeling input parameter, and would be a consequent next step in the image-based wettability characterization and digital rocks analysis. 


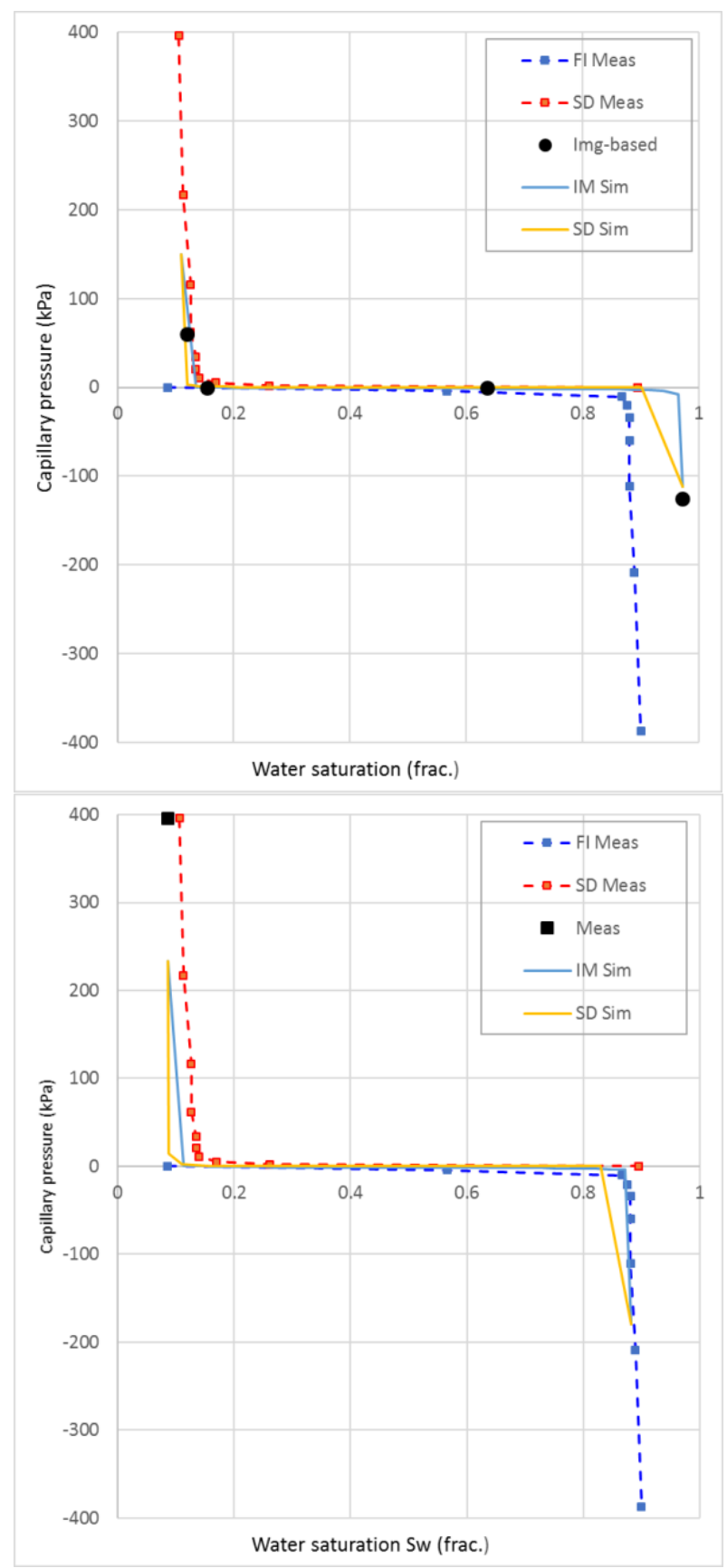

Fig. 4. Digital rocks simulations matching Amott test results for image-based wettability study (top) and for traditional laboratory Amott test on nearby core plugs (bottom). Corresponding Amott and USBM wettability indices are reported in Table 2.

\section{Conclusions}

This study investigated the wettability characterization of a poorly consolidated, high permeable sandstone. Conventional laboratory testing procedures try to limit sample handling, such as moving samples to an Amott cell for spontaneous imbibition/drainage, to avoid damage. We applied a complementary image-based workflow on a smaller sub-plug, with the use of Amott cells for such samples and allowing the use of crude oil throughout the testing cycle.
Findings of this comparison are as follows:

- The presented image-based wettability study confirmed a mixed wet to slightly oil-wet condition of the reservoir, with low spontaneous imbibition of water and significant spontaneous secondary drainage of oil, and according water and oil wetting indices.

- Digital rocks analysis can give additional insight from direct saturation determination in a centrifuge Amott-test procedure, avoiding average or end-face saturation estimation, as well as extension of results to well-anchored dynamic flow functions for reservoir simulation.

- 3D imaging techniques enable the direct visualization and image-based, quantitative characterization at the pore scale, adding value to traditional laboratory measurement procedures by direct observations. The envisioned goal is to establish a new workflow for fluid imaging during laboratory wettability testing under in-situ reservoir conditions.

\section{References}

1. Amott, E., "Observations Relating to the Wettability of Porous Rock," Trans. AIME (1959), 216, 156-162.

2. Donaldson, E.C., Thomas, R.D., and Lorenz, P.B., "Wettability Determination and Its Effect on Recovery Efficiency," SPE J. (1969), 9, 1320.

3. Strand, S., Standnes, D.C., and Austad, T., "New wettability test for chalk based on chromatographic separation of $\mathrm{SCN}^{-}$and SO42-," J. Petr. Sci. and Eng. (2006), 52, 187197.

4. Hirasaki, G. J., "Wettability: Fundamentals and Surface Forces," SPE Formation Eval. (1991), 6, 217-226.

5. Sohrabi, M., Mahzari, P., Farzaneh, S.A., Mills, J.R., Tsolis, P., and Ireland, S., "Novel Insights into Mechanisms of Oil Recovery by Low Salinity Water Injection." SPE-172778-MS (2015).

6. Kumar, M., Fogden, A. Senden, T., and Knackstedt, M., "Investigation of Pore-Scale Mixed Wettability," SPE J. 17, 20-30 (2012).

7. Idowu, N.A., Long, H., Øren, P.E., Carnerup, A.M., Fogden, A., Bondino, I., and Sundal, L., "Wettability analysis using micro-CT, FESEM and QEMSCAN, and its applications to digital rock physics," Int. Symp. Society of Core Analysts (2015), SCA2015-10.

8. Rücker, M., Bartels, W.B., Boone, M.A., Bultreys, T., Mahani, H., and Berg, S., "Porescale Processes in Amott Spontaneous Imbibition Tests," EAGE Conference and Exhibition, Paris (2017).

9. Øren, P.E. and Bakke, S., "Reconstruction of Berea sandstone and pore-scale modeling of 
wettability effects,",J. Petr. Sci. and Eng. (2003), 39, 177-199.

10. Dixit, A.B., Buckley, J.S., McDougall, S.R., and Sorbie, K.S., "Core Wettability: Should $\mathrm{I}_{\mathrm{AH}}$ equal I Issm?," Int. Symp. Society of Core Analysts (1998), SCA1998-09.

11. Prodanovic, M., Lindquist, W.B., and Seright, R.S., "Residual Fluid Blobs and Contact Angle Measurements from X-Ray Images of Fluid Displacement." XVI Int. Conf. Comp. Methods in Water Resources (2006), CMWR-XVI.

12. Andrew, M., Bijeljic, B., and Blunt, M.J., "Porescale Contact Angle Measurements at Reservoir Conditions Using X-ray Microtomography," $A d v$. Water Resources (2014), 68, 24-31. 